\title{
Structure Preserving Port-Hamiltonian Discretization of a 1-D Inflatable Space Reflector
}

\author{
T. Voß and J.M.A. Scherpen
}

\begin{abstract}
In this paper we show how to spatially discretize a distributed port-Hamiltonian ( $\mathrm{pH}$ ) system, which describes the dynamics of an 1-D piezoelectric Euler-Bernoulli beam. Standard spatial discretization schemes for PDE systems have the disadvantage that they typically lead to a finite dimensional system which is not anymore in the $\mathrm{pH}$ form. So, there is a need for a spatial discretization scheme which preserves the structure of the system. The problem of spatially discretizing a pH system with constant Stokes-Dirac structures and quadratic energy functions was solved in the past. But here we consider a piezoelectric Euler-Bernouli with nonlinear deformation. So, the Stokes-Dirac structure and energy function of the system are also nonlinear, and this causes some additional problems.
\end{abstract}

\section{INTRODUCTION}

Inflatable structures are a very promising technology for space applications [1]. Due to the fact that any inflatable structure is built of a polymer casing which is folded on Earth and then inflated with a gas in space, it is clear that an inflatable structure cannot have the same surface accuracy as a rigid body. One way to increase the surface accuracy of an inflatable is to use active control, where the actuators are made of piezoelectric polymers [2].

In this paper we show how to spatially discretize an infinite dimensional port-Hamiltonian $(\mathrm{pH})$ system. The $\mathrm{pH}$ model was obtained by reducing a 3-D continuum mechanics model to an 1-D pH system, see [3]. The spatial discretization of a infinite dimensional system cannot be done by standard discretization schemes, e.g. FEM [4], because these schemes destroy the structure of the $\mathrm{pH}$ system. So, there is a need for a specialized method which preserves the structure of the system. In [5] it is described how to spatially discretize a finite dimensional $\mathrm{pH}$ system with a constant StokesDirac structure and a quadratic energy function by using specialized basis functions to approximate the dynamics.

We consider in this paper a piezoelectric Euler-Bernoulli beam with nonlinear deformation for which the Stokes-Dirac structure and energy function are also nonlinear. The nonlinearity yields additional complications during the spatial discretization. Due to the nonlinearity introduced above, one needs more specialized basis functions to preserve the $\mathrm{pH}$ structure.

The scheme is divided in three main steps. First we define the basis functions, Section III-A, and then approximate the interconnection structure which yields a finite dimensional Dirac structure, Section III. Because the Stokes-Dirac structure is nonlinear, additional constraints for the basis

T.Voss and J.M.A. Scherpen are with Faculty of Mathematics \& Natural science, DTPA Group, University of Groningen, Nijenborgh 4, 9747 AG Groningen, NL T.Vosserug.nl, J.M.A.Scherpen@rug.nl functions will emerge. The second step is to approximate the energy function, Section III-F. This results then in a finite dimensional system which can be used for control design.

\section{THE TO DISCRETIZED SYSTEM AND DISCRETIZATION GOALS}

In this section we describe the infinite dimensional $\mathrm{pH}$ model of a piezoelectric composite which we want to spatially discretize.

\section{A. Infinite dimensional model of a piezoelectric composite}

In this section we define a system that describes the dynamics of a piezoelectric composite in distributed $\mathrm{pH}$ form, for more details see [6], [3]. The composite consists of a base layer (identified by the subscript "b") to which a piezoelectric layer (subscript "p") is bonded. The

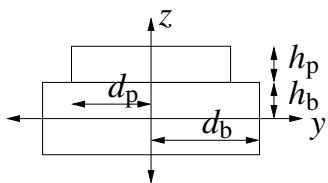

Fig. 1. Cross sectional area of the composite geometry of the system is defined as in Figure 1. We assume that the base layer has a constant thickness $\left(2 d_{\mathrm{b}}\right)$ and a constant height $2 h_{\mathrm{b}}$ while the length is $L$. We define the origin of the $y z$-plane in the center of mass of the base layer. So, the cross sectional area of the base layer $A_{\mathrm{b}}$ is $\left[-d_{\mathrm{b}}, d_{\mathrm{b}}\right] \times\left[-h_{\mathrm{b}}, h_{\mathrm{b}}\right]$. We define $I_{\mathrm{b}, 0}=\int_{A_{\mathrm{b}}} z d A_{\mathrm{b}}=0$ and $I_{\mathrm{b}}=\int_{A_{\mathrm{b}}} z^{2} A_{\mathrm{b}}$. On top of the base layer the piezoelectric layer is bonded. The height of the layer is $h_{\mathrm{p}}$ and the width is given as $2 d_{\mathrm{p}}$. We also assume that the width is symmetric with the $x$-axis. Hence, the cross sectional area of the piezo layer is $A_{\mathrm{p}}$ is $\left[-d_{\mathrm{p}}, d_{\mathrm{p}}\right] \times\left[h_{\mathrm{b}}, h_{\mathrm{b}}+h_{\mathrm{p}}\right]$. We define $I_{\mathrm{p}, 0}=\int_{A_{\mathrm{p}}} z d A_{\mathrm{p}}$ and $I_{\mathrm{p}}=\int_{A_{\mathrm{p}}} z^{2} A_{\mathrm{p}}$. To simplify the notation in the following paragraphs we define $A_{\text {tot }}=A_{\mathrm{b}}+A_{\mathrm{p}}$.

The energy stored in the composite is the sum of the energies stored in the different layers and it can be stated as

$$
H_{\mathrm{tot}}=H_{\mathrm{b}}+H_{\mathrm{p}}=K_{\mathrm{tot}}+P_{\mathrm{tot}}
$$

where $K_{\text {tot }}$ is the total kinetic energy and $P_{\text {tot }}$ is the total potential energy stored in the system. So, we obtain

$$
\begin{aligned}
K_{\mathrm{tot}}= & \frac{1}{2} \int_{0}^{L} \mathbf{p}^{\top} \mathbf{M}_{\mathrm{tot}}^{-1} \mathbf{p} d x \\
P_{\mathrm{tot}}= & \int_{0}^{L} \int_{A_{\mathrm{tot}}} C_{\mathrm{tot}}^{E}\left(u_{0}^{\prime}-z \phi^{\prime}+\frac{1}{2}\left(u_{0}^{\prime}-z \phi^{\prime}\right)^{2}\right. \\
& \left.+\frac{1}{2} \phi^{2}\right)^{2} d A_{\mathrm{tot}}+e A_{\mathrm{p}} E^{2} d x,
\end{aligned}
$$


with

$$
\begin{aligned}
\mathbf{p} & =\mathbf{M}_{\mathrm{tot}} \dot{\mathbf{u}}, \mathbf{M}_{\mathrm{tot}}=\mathbf{M}_{\mathrm{b}}+\mathbf{M}_{\mathrm{p}}, \\
\mathbf{u} & =\left(u_{0}, w, \phi\right)^{\top} \\
C_{\mathrm{tot}}^{E}(z) & =\left\{\begin{array}{cc}
C_{\mathrm{b}}^{E} & \text { for all } z \in\left[-h_{\mathrm{b}}, h_{\mathrm{b}}\right] \\
C_{\mathrm{p}}^{E} & \text { for all } z \in\left(h_{\mathrm{b}}, h_{\mathrm{b}}+h_{\mathrm{p}}\right]
\end{array}\right.
\end{aligned}
$$

here $u_{0}$ and $w$ are the displacements in the $x$ and $z$ direction, respectively, $\phi$ is the rotation of the cross-sectional area and it holds that $\phi=w^{\prime}, M_{\text {tot }}$ is the mass matrix of the systems, $C_{\text {tot }}^{E}$ is the Young's modulus of the used materials, and $E$ is the applied electrical field. All variables depend on the $x$ coordinate and the time $t$. We also define the prime operator as $f^{\prime}=\frac{\partial}{\partial x} f$.

As state variables of the $\mathrm{pH}$ system we choose $\left(\mathbf{p}, \mathbf{u}^{\prime}, E\right)^{\top}$. So, the variational derivative of the Hamiltonian with respect to these state variables is given as

$$
\left[\begin{array}{c}
\delta_{p_{1}} H \\
\delta_{p_{2}} H \\
\delta_{p_{3}} H \\
\delta_{u_{0}^{\prime}} H \\
\delta_{\phi} H \\
\delta_{\phi^{\prime}} H \\
\delta_{E} H
\end{array}\right]=\left[\begin{array}{c}
\dot{u}_{0} \\
\dot{w} \\
\dot{\phi} \\
\int_{A_{\mathrm{tot}}} C_{\mathrm{tot}}^{E} \varepsilon\left(1+u_{0}^{\prime}-z \phi^{\prime}\right) d A_{\mathrm{tot}} \\
\int_{A_{\mathrm{tot}}} C_{\mathrm{tot}}^{E} \varepsilon \phi d A_{\mathrm{tot}} \\
\int_{A_{\mathrm{tot}}} C_{\mathrm{tot}}^{E} \varepsilon\left(-z-z u_{0}^{\prime}+z^{2} \phi^{\prime}\right) d A_{\mathrm{tot}} \\
\varepsilon^{e} A_{\mathrm{e}} E
\end{array}\right]
$$

where

$$
\varepsilon=u_{0}^{\prime}-z \phi^{\prime}+\frac{1}{2}\left(u_{0}^{\prime}-z \phi^{\prime}\right)^{2}+\frac{1}{2} \phi^{2} .
$$

Next we want to state the interconnection structure of the system which describes the energy flow in the system. We will do this in a differential geometric formulation. To simplify the notation we write the system in effort-flow notation.

$$
\begin{aligned}
& \mathbf{e}=\mathbf{J e}+\mathbf{B}\left[\begin{array}{c}
\mathbf{f}_{u} \\
\mathbf{f}_{w} \\
I_{e}
\end{array}\right] \\
& \mathbf{y}=\mathbf{B}^{\top} \mathbf{e},
\end{aligned}
$$

where

$$
\begin{aligned}
& \mathbf{f}=\left[f^{p_{i}}, f^{\varepsilon_{i}}, f^{E}\right]^{\top}=\dot{\mathbf{x}}, \mathbf{e}=\left[e^{p_{i}}, e^{\varepsilon_{i}}, e^{E}\right]^{\top}=\delta_{\mathbf{x}} H \\
& \mathbf{J}=\left[\begin{array}{ccc}
0 & d \mathbf{I}_{3} & \mathbf{g} \\
d \mathbf{I}_{3} & 0 & 0 \\
-\mathbf{g}^{*} & 0 & 0
\end{array}\right], \mathbf{g}=\left[g_{1}(\circ), g_{2}(\circ), g_{3}(\circ)\right]^{\top} \\
& \mathbf{B}^{\top}=\left[\begin{array}{ccccccc}
2 d_{\mathrm{b}} & 0 & -2 d_{\mathrm{b}} h_{\mathrm{b}} & 0 & 0 & 0 & 0 \\
0 & 2 d_{\mathrm{b}} & 0 & 0 & 0 & 0 & 0 \\
0 & 0 & 0 & 0 & 0 & 0 & \frac{1}{\varepsilon^{e} A_{\mathrm{e}}}
\end{array}\right] \\
& g_{1}(\circ)=-\frac{e}{\varepsilon^{e} A_{\mathrm{p}}} d\left(\left(A_{\mathrm{p}}+A_{\mathrm{p}} * u_{0}^{\prime}-I_{\mathrm{p}, 0} * \phi^{\prime}\right) \wedge \circ\right) \\
& g_{2}(\circ)=-\frac{e}{\varepsilon^{e}} d(* \phi \wedge \circ) \\
& g_{3}(\circ)=\frac{e}{\varepsilon^{e} A_{\mathrm{p}}} d\left(\left(I_{\mathrm{p}, 0}+I_{\mathrm{p}, 0} * u_{0}^{\prime}-I_{\mathrm{p}} * \phi^{\prime}\right) \wedge \circ\right)
\end{aligned}
$$

Here $A_{\mathrm{e}}$ is the electrode input area, $*$ is the Hodgestar operator and $\wedge$ is the wedge product. The boundary conditions are given as $e_{B}=\left.e^{\varepsilon}\right|_{\partial Z}, f_{B}=\left.e^{\mathbf{p}}\right|_{\partial Z}$ where $Z=$ $[0, L]$. Physically $e_{B}$ is the force and $f_{B}$ is the velocity at the boundaries. We assume that the boundaries of the beam are clamped, so $f_{B}=0$.
Equation (1) defines a Stokes-Dirac structure if the matrix $J$ is skew-adjoint. But since the operator $d$ is skew-adjoint $(* d=-d)$ it is easy to see that the matrix $J$ is in fact skewadjoint, see [7] for more details. So, the system is a valid distributed $\mathrm{pH}$ system.

\section{DisCRETIZATION OF THE DISTRIBUTED MODEL}

In this section we show how to adapt the discretization method proposed by [5] to a piezoelectric Euler-Bernoulli beam. The goal of this section is to derive a finite $\mathrm{pH}$ model [8] of the piezoelectric beam. To achieve the discretized dynamics of a beam with length $L$ we have to perform several steps. First we have to subdivide the interval $[0, L]$ into $n$ elements. On these elements, e.g. $[a, b]$, we discretize the dynamics. We start with the discretization of the interconnection structure and then discretize the energy function of the system. Next we interconnect the $n$ different finite dimensional $\mathrm{pH}$ models to achieve a lumped model. The interconnection of the $n$ local lumped models approximates then the dynamics of the infinite dimensional model. This discretization approach preserves the $\mathrm{pH}$ structure of the system.

We consider here a part of the piezoelectric composite between two points $a$ and $b(0 \leq a<b \leq L)$. So, the spatial manifold where we want to discretize the system is $Z_{a b}=$ $[a, b]$. Note that all flows of the system are distributions (oneforms), e.g. we cannot define the strain at a certain point, but we can define the strain of an interval $Z$ if we integrate the strain over $Z$. So, the strain in $x$-direction between the points $a$ and $b$ is given as $\int_{Z_{a b}} \varepsilon_{1}(x)$. All efforts are given as functions of position e.g. We can define the velocity of a certain point. So, the velocity can be given as a function $\dot{u}_{0}(x)$.

\section{A. Approximation of efforts and flows}

Similar to the classical finite element approach we define basis functions $\omega$ which are used to approximate the energy and coenergy variables of the system. For every finite element we have a set of basis functions. These basis functions have to fulfill certain constraints, e.g. $\omega_{i}$ has to have the value 1 at one specific edge $\left(x_{i}\right)$ of the finite element

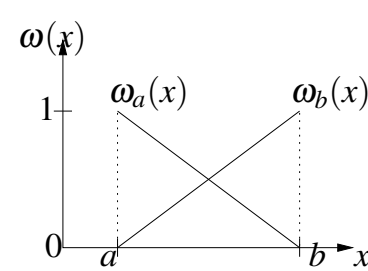

Fig. 2. Example for basis function of a 1-D finite element while being zero at all other edges $\left(x_{j}, j \neq i\right)$, see Figure 2. Additionally the basis functions have to be chosen in a way so that they preserve the $\mathrm{pH}$ structure. The actual approximation consists then of a finite sum of all basis functions defined for that specific finite element each multiplied with a scalar, e.g. $f(x, t) \approx \sum_{i=1}^{n} c_{i}(t) \omega_{i}(x)$. We have to stress here that the scalar is only depending on time and the basis function depends only on the spatial coordinates. The value of each of these scalars has to be chosen in such a way that the approximation is exact at the edges of the finite element, e.g. $f\left(x_{j}, t\right)=\sum_{i=1}^{n} c_{i}(t) \omega_{i}\left(x_{j}\right)$, hence, $f\left(x_{j}, t\right)=c_{j}(t)$. 
Here all flows are one-forms so the basis functions which we use for approximation also have to be one-forms. The efforts, which are zero-forms, can be approximated by zeroforms. The approximation of the zero-forms has to be done in such a way that we have a perfect matching at the boundaries of $Z_{a b}$. Hence, we have to approximate the zeroforms with the sum of two basis function, e.g. the stress in x-direction $\left(\nabla_{u_{0}^{\prime}} H\right)$ in the interval $Z_{a b}$ can be approximated by $e^{\varepsilon_{1}}(x, t) \approx e_{a}^{\varepsilon_{1}}(t) \omega_{a}^{\varepsilon_{1}}(x)+e_{b}^{\varepsilon_{1}}(t) \omega_{b}^{\varepsilon_{1}}(x)$. One possibility for the basis functions is to choose them such that $\omega_{a}(a)=$ $\omega_{b}(b)=1$, and $\omega_{a}(b)=\omega_{b}(a)=0$, because then it holds that $e_{a}(t)=e(a, t)$ and $e_{b}(t)=e(b, t)$. For the approximation of the one-forms we have to assure that the integral over $Z_{a b}$ of the approximated term and the exact term have to coincide, e.g. $\int_{Z_{a b}} f^{p_{i}}(t, x)=\int_{Z_{a b}} f_{a b}^{p_{i}}(t) \omega_{a b}^{p_{i}}(x)$. So, one way is to choose the basis function $\omega_{a b}(x)$ such that $\int_{Z_{a b}} \omega_{a b}(x)=1$, because then we have $f_{a b}^{p_{i}}(t)=\int_{Z_{a b}} f^{p_{i}}(x, t)$.

So, we choose the following approximations of the state variables.

One-forms:

$$
\begin{aligned}
f^{p_{i}}(x, t) & \approx f_{a b}^{p_{i}}(t) \omega_{a b}^{p_{i}}(x), f^{\varepsilon_{i}}(x, t) \approx f_{a b}^{\varepsilon_{i}}(t) \omega_{a b}^{\varepsilon_{i}}(x) \\
f^{E}(x, t) & \approx f_{a b}^{E}(t) \omega_{a b}^{E}(x), \int_{Z_{a b}} \omega_{a b}=1
\end{aligned}
$$

Zero-forms:

$$
\begin{aligned}
e^{p_{i}}(x, t) & \approx e_{a}^{p_{i}}(t) \omega_{a}^{p_{i}}(x)+e_{b}^{p_{i}}(t) \omega_{b}^{p_{i}}(x) \\
e^{\varepsilon_{i}}(x, t) & \approx e_{a}^{\varepsilon_{i}}(t) \omega_{a}^{\varepsilon_{i}}(x)+e_{b}^{\varepsilon_{i}}(t) \omega_{b}^{\varepsilon_{i}}(x) \\
e^{E}(x, t) & \approx e_{a}^{E}(t) \omega_{a}^{E}(x), \omega_{a}(a)=\omega_{b}(b)=1 \\
\omega_{a}(b) & =\omega_{b}(a)=0
\end{aligned}
$$

This is the first set of constraints which the basis functions have to fulfill. Additional constraints come up during the discretization procedure. In order to simplify the notation we omit the time and spatial dependency for all the functions in the following sections.

Due to the fact that the states are directly related to the energy variables $(\dot{\mathbf{x}}=\mathbf{e})$ we can approximate them by using the same one-forms as for the flows

$$
p_{i}=p_{a b}^{i} \omega_{a b}^{p_{i}}, \varepsilon_{i}=\varepsilon_{a b}^{i} \omega_{a b}^{\varepsilon_{i}}, E=E_{a b} \omega_{a b}^{E} .
$$

Note that the following must hold

$$
\left[\begin{array}{c}
f_{a b}^{p_{i}} \\
f_{a b}^{\varepsilon_{i}} \\
f_{a b}^{E}
\end{array}\right]=\frac{\partial}{\partial t}\left[\begin{array}{c}
p_{a b}^{i} \\
\varepsilon_{a b}^{i} \\
E_{a b}
\end{array}\right] .
$$

Using the definitions of one/zero-forms for the energy and co-energy variables we can approximate the equations of motion (1). As example we show how one can approximate the first equation of motion, i.e.

$$
f_{a b}^{p_{1}} \omega_{a b}^{p_{1}}=e_{a}^{\varepsilon_{1}} d \omega_{a}^{\varepsilon_{1}}+e_{b}^{\varepsilon_{1}} d \omega_{b}^{\varepsilon_{1}}+g_{1}\left(e_{a}^{E} \omega_{a}^{E}\right)
$$

Approximating the equations of motions is the first step in deriving a finite dimensional approximation for the infinite dimensional system.

\section{B. Discretization of the interconnection structure}

In this section we show how to derive a finite dimensional representation from the approximation of the equations of motions. To this aim we choose the one and zero-forms in such a way that for every $e_{a}^{i}, e_{b}^{i}$ we can find a $f_{a b}^{i}$. This has to be done such that the $\mathrm{pH}$ structure of the system is preserved. To do this one can assume that every $e_{a / b}^{i}$ is zero except one and calculate the relation between $f_{a b}^{i}$ and $e_{a / b}^{i}$. We take as example the first equation of motion (5).

a) Discretization procedure:

- Assume $e_{b}^{\varepsilon_{1}}=e_{a}^{E}=0$ on $Z_{a b}$. Then it holds that

$$
f_{a b}^{p_{1}} \omega_{a b}^{p_{1}}=e_{a}^{\varepsilon_{1}} d \omega_{a}^{\varepsilon_{1}} .
$$

Next, if $c_{1} f_{a b}^{p_{1}}=e_{a}^{\varepsilon_{1}}$ we can reformulate this as

$$
c_{1} \omega_{a b}^{p_{1}}=d \omega_{a}^{\varepsilon_{1}} .
$$

Now we integrate over $Z_{a b}$ and obtain

$$
c_{1} \underbrace{\int_{Z_{a b}} \omega_{a b}^{p_{1}}}_{=1}=\underbrace{\omega_{a}^{\varepsilon_{1}}(b)}_{=0}-\underbrace{\omega_{a}^{\varepsilon_{1}}(a)}_{=1} .
$$

Since we assumed that $\omega_{a}^{i}(b)=0$ and $\int_{Z_{a b}} \omega_{a b}^{i}=$ $\omega_{a}^{i}(a)=1$ it follows that $c_{1}=-1$. Hence,

$$
-\omega_{a b}^{p_{1}}=d \omega_{a}^{\varepsilon_{1}} \text {. }
$$

Note that the zero-from $\omega_{a}^{\varepsilon_{1}}$ is completely determined by the one-from $\omega_{a b}^{p_{1}}$.

- Assume $e_{a}^{\varepsilon_{1}}=e_{a}^{E}=0$. Then obtains

$$
\omega_{a b}^{p_{1}}=d \omega_{b}^{\varepsilon_{1}}
$$

- Assume $e_{a}^{\varepsilon_{1}}=e_{b}^{\varepsilon_{1}}=0$, then

$$
\begin{aligned}
f_{a b}^{p_{1}} \omega_{a b}^{p_{1}}= & e_{a}^{E} g_{1}\left(\omega_{a}^{E}\right)=-\frac{e}{\varepsilon^{e}} e_{a}^{E} d \omega_{a}^{E} \\
& -\frac{e}{\varepsilon^{e}} e_{a}^{E} u_{a b}^{\prime} d\left(* \omega_{a b}^{\varepsilon_{1}} \wedge \omega_{a}^{E}\right) \\
& +\frac{I_{0}}{A} \frac{e}{\varepsilon^{e}} e_{a}^{E} \phi_{a b}^{\prime} d\left(* \omega_{a b}^{\varepsilon_{3}} \wedge \omega_{a}^{E}\right) .
\end{aligned}
$$

If we now combine all these relations, integrate over $Z_{a b}$ and use the fact that $\int_{Z_{a b}} \omega_{a b}^{p_{1}}=1$ we obtainment

$$
\begin{aligned}
f_{a b}^{p_{1}}= & \left(e_{b}^{\varepsilon_{1}}-e_{a}^{\varepsilon_{1}}\right)-e_{a}^{E} \frac{e}{\varepsilon^{e}}\left(\left.\omega_{a}^{E}\right|_{a} ^{b}-\left.u_{a b}^{\prime}\left(* \omega_{a b}^{\varepsilon_{1}} \wedge \omega_{a}^{E}\right)\right|_{a} ^{b}\right. \\
& \left.+\left.\frac{I_{0}}{A} \phi_{a b}^{\prime}\left(* \omega_{a b}^{\varepsilon_{3}} \wedge \omega_{a}^{E}\right)\right|_{a} ^{b}\right) .
\end{aligned}
$$

For all other equations of motions a similar procedure has to be applied. The simplification of the mechanical part works out in the same way. For the electrical equations of motion we have to discretize $f_{a b}^{E} \omega_{a b}^{E}=\sum_{i=1}^{3} g_{i}\left(e_{a}^{p_{i}} \omega_{a}^{p_{i}}+e_{b}^{p_{i}} \omega_{b}^{p_{i}}\right)$. As example we show here only how this can be done when all $e_{a / b}^{p_{i}}$ are zero except $e_{a}^{p_{1}}$. Hence,

$$
\begin{aligned}
f_{a b}^{E} \omega_{a b}^{E}= & \frac{e}{\varepsilon^{e}} e_{a}^{p_{1}} d \omega_{a}^{p_{1}}+\frac{e}{\varepsilon^{e}} e_{a}^{p_{1}} u_{a b}^{\prime}\left(* \omega_{a b}^{\varepsilon_{1}} \wedge d \omega_{a}^{p_{1}}\right) \\
& -\frac{I_{0}}{A} \frac{e}{\varepsilon^{e}} e_{a}^{p_{1}} \phi_{a b}^{\prime}\left(* \omega_{a b}^{\varepsilon_{3}} \wedge d \omega_{a}^{p_{1}}\right) \\
= & -\frac{e}{\varepsilon^{e}} e_{a}^{p_{1}} \omega_{a b}^{\varepsilon_{1}}-\frac{e}{\varepsilon^{e}} e_{a}^{p_{1}} u_{a b}^{\prime}\left(* \omega_{a b}^{\varepsilon_{1}} \wedge \omega_{a b}^{\varepsilon_{1}}\right) \\
& +\frac{I_{0}}{A} \frac{e}{\varepsilon^{e}} e_{a}^{p_{1}} \phi_{a b}^{\prime} d\left(* \omega_{a b}^{\varepsilon_{3}} \wedge \omega_{a b}^{\varepsilon_{1}}\right)
\end{aligned}
$$




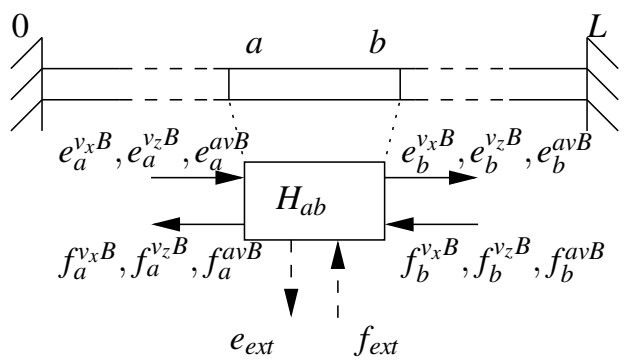

Fig. 3. Boundary ports of a finite element

In this approximation we used that $\omega_{a b}^{p_{i}}=-\mathrm{d} \omega_{a}^{\varepsilon_{i}}$.

To ensure that the approximation of $\mathbf{g}$ fulfills $\mathbf{g}=-\mathbf{g}^{*}$ we have to assume that the basis functions fulfill additional constraints given by

$$
\begin{aligned}
& \left.\left(* \omega_{a b}^{\varepsilon_{i}} \wedge \omega_{a}^{E}\right)\right|_{a} ^{b}=\left.\int_{Z_{a b}} * \omega_{a b}^{\varepsilon_{i}} \wedge \omega_{a b}^{\varepsilon_{i}} \omega_{a}^{E}\right|_{a} ^{b}=1 \\
& \left.\left(* \omega_{a b}^{\varepsilon_{i}} \wedge \omega_{a}^{E}\right)\right|_{a} ^{b}=\int_{Z_{a b}} * \omega_{a b}^{\varepsilon_{i}} \wedge \omega_{a b}^{\varepsilon_{j}}, i, j \in\{1,3\}, i \neq j .
\end{aligned}
$$

The calculations here shown yield then the following finite dimensional approximation of the infinite dimensional equations of motion

$$
\begin{aligned}
f_{a b}^{p_{i}} & =e_{b}^{\varepsilon_{i}}-e_{a}^{\varepsilon_{i}}+c_{i} e_{a}^{E} \\
f_{a b}^{\varepsilon_{i}} & =e_{b}^{p_{i}}-e_{a}^{p_{i}} \\
f_{a b}^{E} & =\sum_{i=1}^{3} c_{i}\left(e_{b}^{p_{i}}-e_{a}^{p_{i}}\right)
\end{aligned}
$$

where the $c_{i}$ for $i=1,2,3$ is the approximation of $g_{i}$ being given by

$$
\begin{aligned}
c_{1} & =-\frac{e}{\varepsilon^{e}}\left(1+u_{a b}^{\prime} \int_{Z_{a b}} * \omega_{a b}^{\varepsilon_{1}} \wedge \omega_{a b}^{\varepsilon_{1}}-\frac{I_{0}}{A} \phi_{a b}^{\prime} \int_{Z_{a b}} * \omega_{a b}^{\varepsilon_{3}} \wedge \omega_{a b}^{\varepsilon_{1}}\right) \\
c_{2} & =-\frac{e}{\varepsilon^{e}} \phi_{a b} \int_{Z_{a b}} * \omega_{a b}^{\varepsilon_{2}} \wedge \omega_{a b}^{\varepsilon_{2}} \\
c_{3} & =\frac{e I_{0}}{\varepsilon^{e} A}\left(1+u_{a b}^{\prime} \int_{Z_{a b}} * \omega_{a b}^{\varepsilon_{1}} \wedge \omega_{a b}^{\varepsilon_{1}}-\frac{I}{I_{0}} \phi_{a b}^{\prime} \int_{Z_{a b}} * \omega_{a b}^{\varepsilon_{3}} \wedge \omega_{a b}^{\varepsilon_{1}}\right)
\end{aligned}
$$

This system is not yet in $\mathrm{pH}$ form because we have not defined any ports yet. To achieve this in the next sections we will define the energy exchange with the neighboring $\mathrm{pH}$ systems and by doing so also automatically define the input/output behavior of the system.

\section{Boundary Variables}

To define the flow of power over the boundaries we have to define relations between the approximation of the co-energy variables and the power ports at the boundaries. In total we have 3 power ports at the boundaries where the system exchanges energy with other neighboring systems, see Figure 3.

1. Velocity/Force in $x$ direction

The first boundary power port consists of the velocity and the force in $x$ direction. The relation between this port and the co-energy variable is given by

$$
\begin{aligned}
F^{x}(a / b) & =e^{\varepsilon_{1}}(a / b)=e_{a / b}^{\varepsilon_{1}}=e_{a / b}^{v_{x} B} \\
v^{x}(a / b) & =e^{p_{1}}(a / b)=e_{a / b}^{p_{1}}=f_{a / b}^{v_{x} B},
\end{aligned}
$$

here $e_{a}^{v_{x} B}$ and $e_{a}^{\nu_{x} B}$ are the force boundary port at the left and right side, respectively. The related velocity ports are expressed by $f_{a / b}^{v_{x} B}$.

2. Velocity/Force in $z$ direction

The second boundary port consists of the velocity and the force in $z$ direction. Here the relation between the boundary port and the co-energy variable can be stated as

$$
\begin{aligned}
F^{z}(a / b) & =e^{\varepsilon_{2}}(a / b)=e_{a / b}^{\varepsilon_{2}}=e_{a / b}^{v_{z} B} \\
v^{z}(a / b) & =e^{p_{2}}(a / b)=e_{a / b}^{p_{2}}=f_{a / b}^{v_{z} B},
\end{aligned}
$$

here $e_{a}^{v_{z} B}$ and $e_{a}^{v_{z} B}$ are the force boundary port at the left and right side, respectively. The related velocity ports are expressed by $f_{a / b}^{v_{x} B}$.

3. Momenta/angular velocity

The last port consists of momenta and angular velocities at the boundaries and is given by

$$
\begin{aligned}
& M_{a / b}=e^{\varepsilon_{3}}(a / b)=e_{a / b}^{\varepsilon_{3}}=e_{a / b}^{a v B} \\
& a v_{a / b}=e^{p_{3}}(a / b)=e_{a / b}^{p_{3}}=f_{a / b}^{a v},
\end{aligned}
$$

where $e_{a}^{a v B}$ and $e_{a}^{a v B}$ are the torque boundary port at the left and right side, respectively. The related angular velocity ports are expressed by $f_{a / b}^{a v B}$.

If we insert these expressions for the boundary ports in (6) and also use the relations between boundary values and coenergy variables (7), (8), and (9) we can define the following relations between the approximation of the efforts $\mathbf{e}_{a}, \mathbf{e}_{b}$ (3) and flows $\mathbf{f}_{a b}$ (2) and the related boundary values $\mathbf{f}^{B}, \mathbf{e}^{B}$.

$$
\left[\begin{array}{c}
\mathbf{f}^{B} \\
\mathbf{e}^{B} \\
\mathbf{f}_{a b}
\end{array}\right]=\left[\begin{array}{ccc}
I_{6} & 0 & 0 \\
0 & I_{6} & 0 \\
0 & K & C_{1} \\
K & 0 & 0 \\
C_{2} & 0 & 0
\end{array}\right] \mathbf{e}_{a / b}
$$

where

$$
\begin{aligned}
& \mathbf{e}^{B}=\left[e_{a}^{v_{x} B}, e_{b}^{v_{x} B}, e_{a}^{v_{z} B}, e_{b}^{v_{z} B}, e_{a}^{a v B}, e_{b}^{a v B}\right]^{\top} \\
& \mathbf{f}_{a b}=\left[f_{a b}^{p_{1}}, f_{a b}^{p_{2}}, f_{a b}^{p_{3}}, f_{a b}^{\varepsilon_{1}}, f_{a b}^{\varepsilon_{2}}, f_{a b}^{\varepsilon_{3}}, f_{a b}^{E}\right]^{\top} \\
& \mathbf{f}^{B}=\left[f_{a}^{v_{x} B}, f_{b}^{v_{x} B}, f_{a}^{v_{z} B}, f_{b}^{v_{z} B}, f_{a}^{a v B}, f_{b}^{a v B}\right]^{\top} \\
& \mathbf{e}_{a / b}=\left[e_{a}^{p_{1}}, e_{b}^{p_{1}}, \cdots, e_{b}^{p_{3}}, e_{a}^{\varepsilon_{1}}, \cdots, e_{b}^{\varepsilon_{3}}, e_{a}^{E}\right]^{\top} \\
& K=\left[\begin{array}{cccccc}
-1 & 1 & 0 & 0 & 0 & 0 \\
0 & 0 & -1 & 1 & 0 & 0 \\
0 & 0 & 0 & 0 & -1 & 1
\end{array}\right] \\
& C_{1}=\left[c_{1}, c_{2}, c_{3}\right]^{\top} \\
& C_{2}=\left[-c_{1}, c_{1},-c_{2}, c_{2},-c_{3}, c_{3}\right]^{\top}
\end{aligned}
$$


These relations will be used in the next section to define the Dirac structure in kernel image representation of the finite dimensional model.

\section{Net power of the system}

In order to define an approximation to the efforts $\mathbf{e}$ on the interval $Z_{a b}$, we first define the power stored in the system. by

The power stored in the system on the interval $Z_{a b}$ is given

$$
\begin{aligned}
P_{a b}^{\mathrm{net}}= & \sum_{i=1}^{3}\left(\int_{Z_{a b}} e^{p_{i}} f^{p_{i}}+\int_{Z_{a b}} e^{\varepsilon_{i}} f^{\varepsilon_{i}}\right)+\int_{Z_{a b}} e^{E} f^{E} \\
& -f_{a}^{v_{x} B} e_{a}^{v_{x} B}+f_{b}^{v_{x} B} e_{b}^{v_{x} B}-f_{a}^{v_{z} B} e_{a}^{v_{z} B}+f_{b}^{v_{z} B} e_{b}^{v_{z} B} \\
& -f_{a}^{a v B} e_{a}^{a v B}+f_{b}^{a v B} e_{b}^{a v B} .
\end{aligned}
$$

If we insert the approximation for the flows (2) and efforts (3) in (11). Then we can reformulate the net power as

$$
P_{a b}^{\mathrm{net}}=\sum_{i=1}^{3}\left(e_{a b}^{p_{i}} f_{a b}^{p_{i}}+e_{a b}^{\varepsilon_{i}} f_{a b}^{\varepsilon_{i}}\right)+e_{a b}^{E} f_{a b}^{E}+P_{a b}^{B}
$$

where we used the following notation

$$
\begin{aligned}
e_{a b}^{i}= & \alpha_{a}^{i} e_{a}^{i}+\alpha_{b}^{i} e_{b}^{i} \\
\alpha_{a}^{i}= & \int_{Z_{a b}} \omega_{a}^{i} \omega_{a b}^{i}, \alpha_{b}^{i}=\int_{Z_{a b}} \omega_{b}^{i} \omega_{a b}^{i} \\
e_{a b}^{E}= & \alpha^{E} e_{a}, \alpha^{E}=\int_{Z_{a b}} \omega_{a}^{E} \omega_{a b}^{E} \\
P_{a b}^{B}= & -f_{a}^{v_{x} B} e_{a}^{v_{x} B}+f_{b}^{v_{x} B} e_{b}^{v_{x} B}-f_{a}^{v_{z} B} e_{a}^{v_{z} B} \\
& +f_{b}^{v_{z} B} e_{b}^{v_{z} B}-f_{a}^{a v B} e_{a}^{a v B}+f_{b}^{a v B} e_{b}^{a v B} .
\end{aligned}
$$

So, we are now able to rewrite the power stored on the interval as a sum over approximated flow times approximated effort as it is also done in finite dimensional $\mathrm{pH}$ modeling.

One way to define a $\mathrm{pH}$ system is the kernel image representation, see [5]. In this representation we reformulate the relations between energy, co-energy, and boundary ports (10) as $E \mathbf{e}+F \mathbf{f}=0$. In this form a boundary port is not explicitly defined but because we have already defined the boundary port we chose here $\mathbf{e}=\left[\mathbf{e}_{a b}, \mathbf{e}^{B}\right]^{\top}$ and $\mathbf{f}=\left[\mathbf{f}_{a b}, \mathbf{f}^{B}\right]^{\top}$. Then $E$ and $F$ are then chosen in such a way that we fulfill the given relations between the boundary ports and co-energy variables (10). Additionally we have to fulfill (6).

$$
\begin{gathered}
{\left[\begin{array}{cccc}
0 & 0 & -\frac{1}{\alpha^{E}} C_{1} & -K \\
0 & 0 & 0 & 0 \\
0 & 0 & 0 & 0 \\
I_{3} & 0 & 0 & 0 \\
0 & I_{3} & 0 & \alpha_{1}
\end{array}\right]\left[\begin{array}{c}
\mathbf{e}_{a b} \\
\mathbf{e}^{B}
\end{array}\right]} \\
+\left[\begin{array}{cccc}
I_{3} & 0 & 0 & 0 \\
0 & I_{3} & 0 & -K \\
0 & 0 & 1 & C \\
0 & 0 & 0 & \alpha_{2} \\
0 & 0 & 0 & 0
\end{array}\right]\left[\begin{array}{c}
\mathbf{f}_{a b} \\
\mathbf{f}^{B}
\end{array}\right]=0
\end{gathered}
$$

where

$$
\begin{aligned}
\mathbf{e}_{a b}= & {\left[e_{a b}^{p_{1}}, e_{a b}^{p_{2}}, e_{a b}^{p_{3}}, e_{a b}^{\varepsilon_{1}}, e_{a b}^{\varepsilon_{2}}, e_{a b}^{\varepsilon_{3}}, e_{a b}^{E}\right]^{\top} } \\
\alpha_{1}= & {\left[\begin{array}{cccccc}
-\alpha_{a}^{\varepsilon_{1}} & -\alpha_{b}^{\varepsilon_{1}} & 0 & 0 & 0 & 0 \\
0 & 0 & -\alpha_{a}^{\varepsilon_{2}} & -\alpha_{b}^{\varepsilon_{2}} & 0 & 0 \\
0 & 0 & 0 & 0 & -\alpha_{a}^{\varepsilon_{3}} & -\alpha_{b}^{\varepsilon_{3}}
\end{array}\right] } \\
\alpha_{2}= & {\left[\begin{array}{cccccc}
-\alpha_{a}^{p_{1}} & -\alpha_{b}^{p_{1}} & 0 & 0 & 0 & 0 \\
0 & 0 & -\alpha_{a}^{p_{2}} & -\alpha_{b}^{p_{2}} & 0 & 0 \\
0 & 0 & 0 & 0 & -\alpha_{a}^{p_{3}} & -\alpha_{b}^{p_{3}}
\end{array}\right] }
\end{aligned}
$$

Equation (12) defines a finite dimensional Dirac structure.

\section{E. Formulation of the interconnection structure}

It can be shown that all representations of $\mathrm{pH}$ systems are equivalent, so, we are able to rewrite the system given in image kernel representation of the last section into the input/output representation. Therefore, we define the following states, inputs, and outputs

$$
\begin{aligned}
\dot{\mathbf{x}}_{a b} & =\mathbf{f}_{a b}, \nabla_{x} H_{a b}=\mathbf{e}_{a b} \\
\mathbf{u}_{a b} & =\left[e_{a}^{v_{x} B}, e_{a}^{v_{z} B}, e_{a}^{a v B}, f_{b}^{v_{x} B}, f_{b}^{v_{z} B}, f_{b}^{a v B}\right]^{\top} \\
\mathbf{y}_{a b} & =\left[-f_{a}^{v_{x} B},-f_{a}^{v_{z} B},-f_{a}^{a v B}, e_{b}^{v_{x} B}, e_{b}^{v_{z} B}, e_{b}^{a v B}\right]^{\top},
\end{aligned}
$$

where $H_{a b}$ is the approximation of the Hamiltonian on $Z_{a b}$ (defined in Section III-F). As for any $\mathrm{pH}$ system the choice of inputs and outputs is arbitrary. To simplify the notation we use the following properties of the basis functions

$$
1+\frac{\alpha_{b}^{i}}{\alpha_{a}^{i}}=\frac{1}{\alpha_{b}^{i}}, 1+\frac{\alpha_{b}^{i}}{\alpha_{a}^{i}}=\frac{1}{\alpha_{a}^{i}}, \alpha_{a}^{\varepsilon_{i}}=\alpha_{b}^{p_{i}}, \alpha_{a}^{p_{i}}=\alpha_{b}^{\varepsilon_{i}} .
$$

Additionally we choose the basis functions such that $\alpha_{a}^{E}=$ $\alpha_{a}^{p_{i}}$.

If we insert (13) in (12) we can write the pH system in input/output form

$$
\begin{aligned}
\dot{\mathbf{x}}_{a b} & =\mathbf{J}_{a b} \nabla_{x} H_{a b}+\mathbf{B}_{a b} \mathbf{u}_{a b} \\
\mathbf{y}_{a b} & =\mathbf{B}_{a b}^{T} \nabla_{x} H_{a b}+\mathbf{D}_{a b} \mathbf{u}_{a b}
\end{aligned}
$$

where

$$
\begin{aligned}
\mathbf{J}_{a b}= & {\left[\begin{array}{ccc}
0 & \mathbf{A} & \frac{C_{1}}{\alpha_{E}} \\
-\mathbf{A} & 0 & 0 \\
-\frac{C_{1}^{T}}{\alpha_{a}^{\rho_{1}}} & 0 & 0
\end{array}\right], \mathbf{A}=\operatorname{diag}\left(\frac{1}{\alpha_{b}^{\varepsilon_{1}}}, \frac{1}{\alpha_{b}^{\varepsilon_{2}}}, \frac{1}{\alpha_{b}^{\varepsilon_{3}}}\right) } \\
\mathbf{B}_{a b}= & {\left[\begin{array}{cc}
-\mathbf{A} & 0 \\
0 & \mathbf{A} \\
0 & C_{1}^{T} \mathbf{A}
\end{array}\right], \mathbf{D}_{a b}=\left[\begin{array}{cc}
0 & -\mathbf{I}_{3} \\
\mathbf{I}_{3} & 0
\end{array}\right] }
\end{aligned}
$$

It is straight forward to see that this finite dimensional interconnection structure is skew symmetric. This is the final discretized interconnection structure. But to be able to define a complete finite dimensional $\mathrm{pH}$ model we additionally have to discretize the energy function of the system.

\section{F. Approximation of the energy function}

The last step to achieve a finite dimensional model of the piezoelectric composite is to discretize the energy function. The Hamiltonian of the system is given as

$$
H=\frac{1}{2} \int_{0}^{L} \mathbf{p}^{T} \mathbf{M}_{t o t}^{-1} \mathbf{p}+\int_{A_{t o t}} \tilde{\varepsilon}^{T} \mathbf{C}(\tilde{\varepsilon}) \tilde{\varepsilon} d A_{t o t}+e A_{p} E^{2} d x
$$




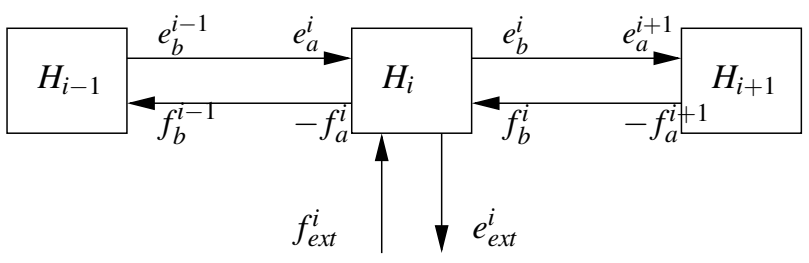

Fig. 4. Interconnection of the $i$-th system with the neighboring systems

where

$$
\begin{aligned}
\mathbf{C}(\tilde{\varepsilon}) & =C_{t o t}^{E} h(\tilde{\varepsilon})^{\top} h(\tilde{\varepsilon}) \\
h(\tilde{\varepsilon}) & =\left(1+\frac{1}{2} u^{\prime}-z \phi^{\prime}, \frac{1}{2} \phi,-z\left(1+\frac{1}{2} u^{\prime}-z \phi^{\prime}\right)\right)
\end{aligned}
$$

Using (4) we can approximate the energy function $H_{a b}$ on the interval $Z_{a b}$ as

$$
H_{a b}=\frac{1}{2} \mathbf{p}_{a b}^{\top} \mathbf{M}_{a b}^{-1} \mathbf{p}_{a b}+\frac{1}{2} \tilde{\varepsilon}_{a b}^{\top} \mathbf{C}_{a b}\left(\tilde{\varepsilon}_{a b}\right) \tilde{\varepsilon}_{a b}+\frac{1}{2} \tilde{e} E_{a b}^{2}
$$

where

$$
\begin{aligned}
\mathbf{p}_{a b} & =\left[\begin{array}{c}
p_{a b}^{1} \\
p_{a b}^{2} \\
p_{a b}^{3}
\end{array}\right], \mathbf{M}_{a b}^{-1}=\int_{Z_{a b}} \Omega_{a b}^{p} \mathbf{M}^{-1} \Omega_{a b}^{p} \\
\tilde{\varepsilon}_{a b} & =\left[\begin{array}{c}
u_{a b}^{\prime} \\
\phi_{a b} \\
\phi_{a b}^{\prime}
\end{array}\right], \tilde{e}=e A \int_{Z_{a b}} * w_{a b}^{E} \wedge w_{a b}^{E} \\
\mathbf{C}_{a b}\left(\tilde{\varepsilon}_{a b}\right) & =\int_{Z_{a b}} \Omega_{a b}^{\varepsilon} \mathbf{C}\left(\Omega_{a b}^{\varepsilon} \tilde{\varepsilon}_{a b}\right) \Omega_{a b}^{\varepsilon} \\
\Omega_{a b}^{p} & =\operatorname{diag}\left(\omega_{a b}^{p_{1}}, \omega_{a b}^{p_{2}}, \omega_{a b}^{p_{3}}\right) \\
\Omega_{a b}^{\varepsilon} & =\operatorname{diag}\left(\omega_{a b}^{\varepsilon_{1}}, \omega_{a b}^{\varepsilon_{2}}, \omega_{a b}^{\varepsilon_{3}}\right)
\end{aligned}
$$

This can then be used to define the values $\mathbf{e}_{a b}$ because it holds that

$$
e_{a b}^{p_{i}}=\frac{\partial H_{a b}}{\partial p_{i}}, e_{a b}^{\varepsilon_{i}}=\frac{\partial H_{a b}}{\partial \varepsilon_{i}}, e_{a b}^{E}=\frac{\partial H_{a b}}{\partial E} .
$$

Substituting $H_{a b}$ in (14) yields a finite dimensional $\mathrm{pH}$ model of a finite element in the interval $Z_{a b}$. This model can then be used to derive a finite dimensional $\mathrm{pH}$ model of a full Euler-Bernoulli beam.

\section{G. Interconnection of the subsystems and simulation results}

With the procedure from the past sections we can calculate $n$ simple finite dimensional $\mathrm{pH}$ systems which describe the dynamics of the beam locally (on the interval $\left[a_{i}, b_{i}\right]$ where it holds that $a_{i}=b_{i-1}$ ). To achieve a global model for the dynamics of the beam we have to interconnect the system in a simple manner. In (13) we have defined the inputs and outputs of a local system. Note that the input for the $i$ th system consists of the efforts at the left boundary and the flows at the right boundary. So, the $i$ th system is interconnected to the $i-1$ st and the $i+1$ st system. More specifically it must hold that $e_{a}^{i}=e_{b}^{i-1}$ and $f_{b}^{i}=f_{a}^{i+1}$. This gives us an interconnection of the $i$ th system with the neighboring systems, as in Figure 4.

The simulation in Matlab shows the behavior of a simple piezoelectric beam. We consider Kapton as material for the base layer, and PVDF as piezoelectric material. The base

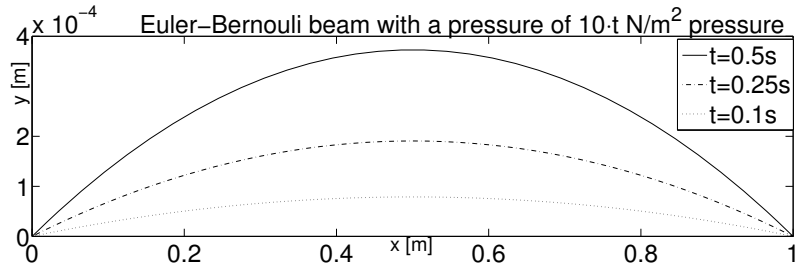

Fig. 5. Simulation of a piezoelectric beam

layer has $1 \mathrm{~m}$ length, $2 \mathrm{~cm}$ thickness and width of $2 \mathrm{~cm}$ . The piezoelectric material covers the whole beam and is $0.25 \mathrm{~cm}$ thick. For the simulation we apply a pressure of $10 \cdot t \frac{\mathrm{N}}{\mathrm{m}^{2}}$ until we reach a pressure of $5 \frac{\mathrm{N}}{\mathrm{m}^{2}}$, see Figure 5 . We show Snapshots at time $t \in\{0.1,0.25,0.5\}$, and it behaves as expected.

\section{CONCLUSION}

In this paper we show how to spatially discretize an infinite dimensional piezoelectric beam in port Hamiltonian $(\mathrm{pH})$ form. The proposed discretization method preserves $\mathrm{pH}$ structure of the system, different to standard discretization methods [4]. The derived model can now be used to design a finite dimensional controller for an 1-D inflatable structure. In future work we will extend these modeling and discretization techniques to 2-D problems.

\section{ACKNOWLEDGMEnTs}

We would like to thank the MicroNed programme for the funding of the research.

\section{REFERENCES}

[1] C. Jenkins, Gossamer Spacecraft: Membrane and Inflatable structures technology for space applications. Progress in Astronautics and Aeronautics, 2001, vol. 191.

[2] A. Vinogradov, S. Ji, C. Jenkins, and Y. Bar-Cohen, "State-of-the-art developments in the field of electro active polymers," in Materials Research Society Fall Meeting, November 2005.

[3] T. Voß, J. Scherpen, and P. Onck, "Modeling for control of an inflatable space reflector, the non-linear 1-d case." CDC, 2008.

[4] O. Zienkiewicz and R. Taylor, The Finite Element Method. McGrawHill Book Company, 1989, vol. 1.

[5] G. Golo, V. Talasila, A. van der Schaft, and B. Maschke, "Hamiltonian discretization of boundary control systems," Automatica, vol. 40, no. 757-771, 2004.

[6] A. Macchelli, "Port hamiltonian systems. a unified approach for modeling and control finite and infinite dimensional physical systems," Ph.D. dissertation, University of Bologna, DEIS, 2003.

[7] B. Maschke and A. J. van der Schaft, "Port controlled hamiltonian representation of distributed parameter sytems," in Proceedings IFAC Workshop on Lagrangian and Hamiltonian Methods for Nonlinear Control, N. Leonard and R. Ortega, Eds., 2000, pp. 28-38.

[8] A. J. van der Schaft and B. M. Maschke, "The Hamiltonian formulation of energy conserving physical systems with external ports," Archiv für Elektronik und Übertragungstechnik, vol. 49, pp. 362-371, 1995. 